\title{
Molecular Docking Studies of Arbutin Derivatives as Tyrosinase Inhibitors
}

\author{
Ayu Masyita ${ }^{1}$, Yusnita Rifai2 ${ }^{*}$ \\ 1 Postgraduate Student, Faculty of Pharmacy, Hasanuddin University, Makassar, Indonesia. \\ 2 Pharmaceutical Chemistry Laboratory, Faculty of Pharmacy, Hasanuddin University, Makassar, Indonesia. \\ * Corresponding author. Tel.: +62 821-9656-2691; email: yusnitarifai@gmail.com \\ Manuscript submitted Decemeber 1, 2018; accepted April 9, 2019.
}

doi: 10.17706/ijbbb.2019.9.3.188-193

\begin{abstract}
Arbutin is a natural skin-whitening agent as a tyrosinase inhibitor. Arbutin could effectively inhibit the activity of tyrosinase in skin cells and block the formation of melanin without affecting cell proliferation. In the development of drugs, such as arbutin derivatives, a simulation of interactions between tyrosinase enzymes as the target proteins with ligands (arbutin derivatives) is required, by using molecular docking simulation method. Preparation of ligands and proteins was performed using AutoDock 4.2. The results show that $\alpha$-arbutin derivatives ( $\alpha$-arbutin-undecylinate ester and deoxy- $\alpha$-arbutin) is able to inhibit a tyrosinase.
\end{abstract}

Key words: Arbutin, molecular docking, tyrosinase inhibitor, hyperpigmentation.

\section{Introduction}

Hyperpigmentation on the epidermis is caused by excessive synthesis of melanin [1], [2]. Biosynthesis of melanin depends on the activity of tyrosinase and ultraviolet radiation (UV) [3]. Tyrosinase is an enzyme that plays an important role in the formation of melanin. The tyrosinase will become L-DOPA, which then, the oxidation of L-DOPA will form dopaquinone (DQ) and DQ undergoes a further transformation, forming melanin [1].

Melanin is responsible for the absorption and protection of skin from harmful UV radiation. The presence of UV radiation will stimulate excessive synthesis of melanin and cause discoloration and the formation of dark spots on the skin [2]. Tyrosinase inhibitors, i.e. hydroquinone, have been widely used in the cosmetic industry as lightening compounds [1]. However, there have been many studies showing the side effects of the hydroquinone. Hydroquinone can result in skin irritation, damage cell membrane lipids, inhibit DNA and RNA synthesis, and contribute to glutathione deficiency. In addition, hydroquinone can stimulate an inflammatory reaction and increase the risk of postinflammatory hyperpigmentation [4].

There are many substances that can be used to inhibit tyrosinase as a skin-whitening or skin-lightening agent, such as arbutin, hydroquinone, vitamin C, kojic acid, linoleic acid, catechol, and hinokitol. But arbutin is the most popular and effective used because of its very low cytotoxicity [3]. Arbutin could accelerate the decomposition and excretion of melanin and thereby reduce skin pigmentation and eliminate freckles. Arbutin has two isoforms, i.e. 4-hydroxyphenyl- $\beta$-glucuronidase $(\beta$-arbutin, Fig. 1 (a) and 4-hydroxyphenyl- $\alpha$-glucuronidase ( $\alpha$-arbutin, Fig. 1(b). Currently, arbutin can be obtained either chemically or enzymatically.

Study on arbutin and its derivatives has been widely conducted [5]. The activity of arbutin and its 
derivatives is related to particle size, spatial structure, and electrostatic potential around the benzene ring. $\alpha$-Arbutin is 10 times as effective in inhibiting tyrosinase as $\beta$-arbutin, but $\alpha$-arbutin is easily degraded by heat [6].

Arbutin $(\beta$-arbutin, molecular weight $=272.25 \mathrm{Da})$ has a highly hydrophilic $(\log \mathrm{P}$ value $=-1.49)$, so that it is relatively low to penetrate into the skin [6]. Arbutin is resistant to light and unstable at pH 2. Arbutin can experience partial hydrolysis in the presence of water into hydroquinone which can be oxidized to benzoquinone.

In modern drug discovery, interaction between a small molecule (ligand) and a protein plays an important role in predicting the orientation of the ligand when it is bound to a protein receptor or enzyme. One of the ways to achieve this condition is by using molecular docking simulation method [7]. Molecular docking is one of the most important tools for drug design that helps prediction of the interaction between macromolecules and ligands, substrates, and inhibitors. In other words, this technique provides an estimate of how well small molecules fit in the active site of a protein [8]. This technique is effective in the discovery of drug candidates before prior to the process of drug synthesis. The benefit of this method is to provide information about the affinity of arbutin derivatives against tyrosinase enzymes.<smiles>OCC1OC(Oc2ccc(O)cc2)C(O)C(O)C1O</smiles>

(a)<smiles>OCC1OC(O)C(O)C(O)C1Oc1ccc(O)cc1</smiles>

(b)<smiles>C1CCCOCCC1</smiles>

(c)<smiles>C=CCCCCCCCCC(=O)OCCOC(O)C1C(O)C(O)C(O)C1Oc1ccc(O)cc1</smiles>

(d)

Fig. 1. The molecular structures of (a) $\beta$-arbutin, (b) $\alpha$-arbutin, (c) deoxy- $\alpha$-arbutin, (d) $\alpha$-arbutin undecylinate ester.

\section{Methods}

\subsection{Preparation of Protein and Ligands}

The molecular structure of mushroom tyrosinase was obtained from Protein Data Bank (PDB ID: 2Y9W, chain A). Small molecules, including water and holmium (Ho) atoms were removed from the target enzyme, excluding copper (II) ions using YASARA ${ }^{\circledR}$ 11.3.2. The 3D structures of $\alpha$-arbutin and $\beta$-arbutin were obtained from PubChem Compound (NCBI) with CID: 158637 and 346. The 3D structures of other arbutin derivatives were made using Marvin Sketch. Preparation of proteins was performed using AutoDock 4.2. Rotatable bonds in the ligands (arbutin derivatives) were assigned by AutoDockTools4 program. Grid parameter files were built using AutoGrid 4.2.6. 


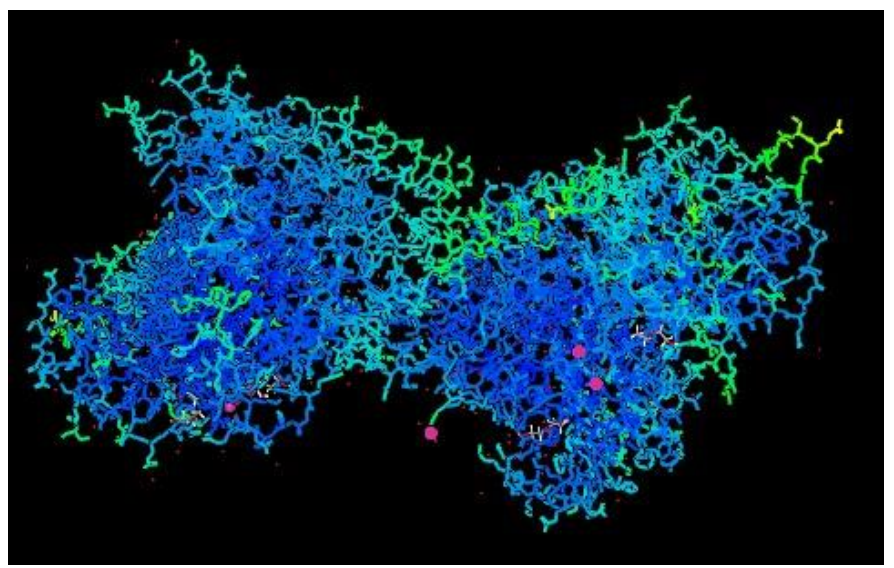

Fig. 2. The molecular structures of mushroom tyrosinase from Agaricus bisporus (PDB ID: 2Y9W).

\subsection{Docking Simulations}

Docking was performed by directing the optimized ligand molecular model on the active site of the tyrosinase using AutoDock Vina [9] for three replications. Then the calculation of the binding of ligand and receptor on the various poses will appear as energy interaction. The protein-ligand interactions were visualized and analyzed using PyMOL(TM) 2.1.1 (Schrodinger, LLC, Cambridge, MA, USA). Molecules with low binding energies show a high degree of stability affinity.

Table 1. The Molecular Interactions of Tyrosinase with Arbutin and its Derivatives

\begin{tabular}{llll}
\hline \hline \multirow{2}{*}{ Compounds } & $\begin{array}{l}\text { Interaction } \\
\text { Energy (Kcal/mol) }\end{array}$ & $\begin{array}{l}\text { H-Bonds } \\
\text { Interaction }\end{array}$ & $\begin{array}{l}\text { Van der Waals } \\
\text { Interaction }\end{array}$ \\
\hline$\beta$-Arbutin & -6.3 & His178, Gln244 & $\mathrm{Cu} 401$ \\
$\alpha$-Arbutin & -6.8 & Asp312, Glu356, Glu359 & $\mathrm{Cu} 401$ \\
$\alpha$-Arbutin-undecylenate ester & -6.6 & Asn174, Lys158 & $\mathrm{Cu} 401$ \\
Deoxy- $\alpha$-arbutin & -6.4 & Thr308 & $\mathrm{Cu} 401$ \\
\hline \hline
\end{tabular}

\section{Results and Discussion}

Molecular docking studies explore the behavior of ligand in the binding site of a target protein. Molecular docking with AutoDock Vina can be prescribed scoring function. The scoring function is used to estimate the tightness of binding and rank docking poses according to their potential binding affinities [10]. This technique is born as a user-friendly software application to perform docking simulations, with high confidence on the results produced and minimal effort for setup and configuration.

In this work, the binding interactions of arbutin and its derivatives were indicative of tyrosinase inhibition activity. Arbutin as tyrosinase inhibitor is hydroquinone derivative named also 4-hydroxyphenyl- $\beta$-glucopyranoside ( $\beta$-arbutin). The inhibition of tyrosinase, decreasing of melanocyte metabolism and avoiding of UV exposure are ways to reduce melanin synthesis. Arbutin showed more effective tyrosinase inhibition than its aglycone, that is hydroquinone. Arbutin has very minor risks and side effects. Arbutin is an effective agent for treatment of hyperpigmentation disorders and demonstrates less melanocyte cytotoxicity than hydroquinone. Arbutin affected skin pigmentation by inhibiting the activity of tyrosinase but without affecting its mRNA expression and synthesis [11]. 


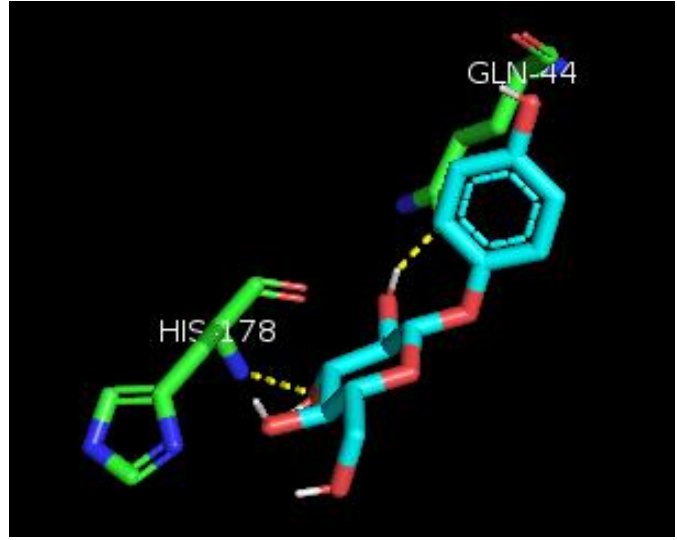

(a)

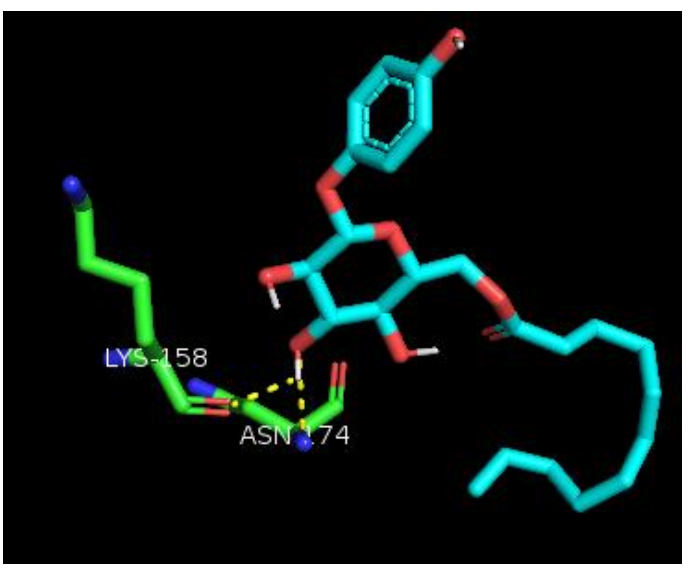

(c)

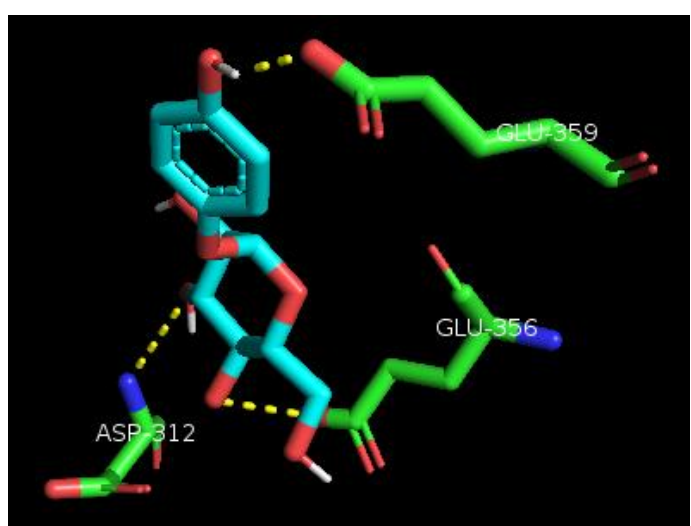

(b)

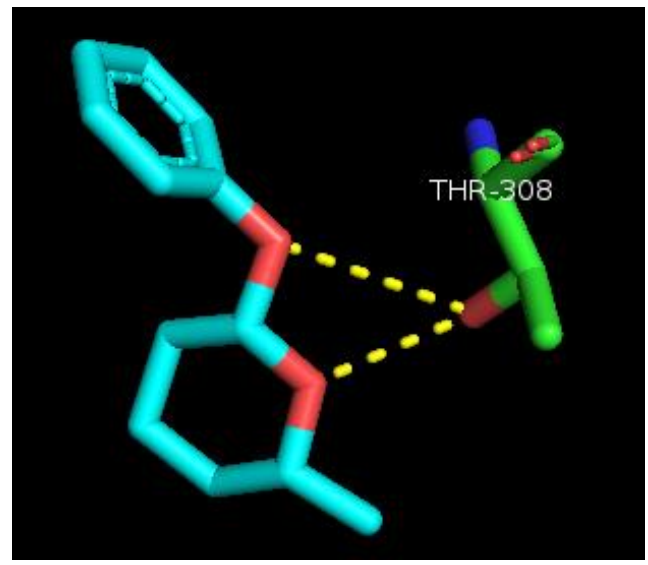

(d)

Fig. 3. Interaction mode of (a) $\beta$-arbutin, (b) $\alpha$-arbutin, (c) deoxy- $\alpha$-arbutin, (d) $\alpha$-arbutin undecylinate ester with tyrosinase. Green stick and blue stick are represented as amino acid residues of tyrosinase and ligands, respectively.

For the purpose of developing a safe and effective skin-whitening agent, the arbutin derivatives are of great candidacy for evaluating its inhibitory activity against mushroom tyrosinase. Research concerning on arbutin derivatives proves them to be more effective than arbutin itself. The results of molecular docking studies show that arbutin derivatives can inhibit tyrosinase, indicated by the interaction energy (docking score). The results indicate that the interaction energy of arbutin derivatives was better than the pure arbutin, in this case, the $\beta$-arbutin.

The compound of $\alpha$-arbutin has the best interaction energy of $-6.8 \mathrm{kcal} / \mathrm{mol}$, while that of $\beta$-arbutin only $-6.3 \mathrm{kcal} / \mathrm{mol}$ (Table 1, Fig. 3). When compared to the other arbutin derivatives, the deoxy- $\alpha$-arbutin compound Fig. 1(c) has nearly the same interaction energy as that of $\beta$-arbutin to bind the active site of tyrosinase, that is $-6.4 \mathrm{kcal} / \mathrm{mol}$ (Table 1, Fig. 3). The $\alpha$-arbutin-undecylenate ester compound Fig. 1(d) has an almost the same interaction energy as that of $\alpha$-arbutin, that is $-6.6 \mathrm{kcal} / \mathrm{mol}$. The interaction of arbutin derivatives with the active site, where it interacts with LYS 158, ASN 174, HIS178, GLN 244, THR 308, GLU 356, GLU 359 and Van der Waals interaction with $\mathrm{Cu} 401$. Docking of $\beta$-arbutin to mushroom tyrosinase has previously been reported to occur at the active site (E256 and N260). The discrepancy with our results is due to the different tyrosinase form used by the previous authors. Unlike their works, we used the deoxy form of tyrosinase where the met form was selected for docking [12].

The similarity of the structure of a compound causes the possibility of binding to the same receptor is 
also greater. The protein-ligand interactions is associated with binding affinity, that is the strength of the compound to bind with the other compounds, that is bind reversibly (interact) [13]. The amount of bonding with the part of hydrophobic can increase the binding affinity, and also inhibition activity of tyrosinase.

Molecular docking studies, in this work, showed accordance with previous studies about arbutin and its derivatives. Deoxy- $\beta$-arbutin showed more effective tyrosinase inhibition than arbutin and hydroquinone [14]. 6-0-undecylenoyl-p-hydroxyphenyl- $\beta$-D-glucopyranoside ( $\beta$-arbutin undecylenate ester) exhibited greater anti-melanogenic activity than arbutin [2]. Also, deoxy-arbutin and its derivatives inhibited both tyrosine hydroxylase, DOPA oxidase activity of tyrosinase and cell proliferation [15]. In another study, $\alpha$-arbutin was stronger than arbutin in inhibiting the activity of tyrosinase [6]. Our study showed $\alpha$-arbutin derivatives: $\alpha$-arbutin-undecylenate ester and deoxy- $\alpha$-arbutin more effective tyrosinase inhibition than $\beta$-arbutin derivatives.

\section{Conclusion}

Arbutin derivatives ( $\alpha$-arbutin-undecylenate ester and deoxy- $\alpha$-arbutin) are of great treatment for pigmentation. They may be developed as cosmetically skin-whitening agents and can be used for further research. Molecular docking using AutoDock program is beneficial to optimize the affinity of ligands that bind to receptors.

\section{Acknowledgment}

This work was supported by Ministry of Science and Technology of Indonesia through grant of PMDSU scholarship Batch III.

\section{References}

[1] Lee, S. Y., Baek, N., \& Nam, T. (2015). Natural, semisynthetic and synthetic tyrosinase inhibitors. Journal of Enzyme Inhibition and Medicinal Chemistry, 31(1), 1-13.

[2] Tokiwa, Y., Kitagawab, M., Raku, T., Yanagitanib, S., \& Yoshinob, K. (2007). Enzymatic synthesis of arbutin undecylenic acid ester and its inhibitory effect on melanin synthesis. Bioorganic \& Medicinal Chemistry Letters, 17(1), 3105-3108.

[3] Garcia-Jimenez, A., Teruel-Puche J. A., Berna, J., Rodriguez-Lopez, J. N., Tudela, J., \& Garcia-Canovas, F. (2017). Action of tyrosinase on alpha and beta-arbutin: A kinetic study. Plos One, 12(5), 1-19.

[4] Reszko, A. E., Berson, D., \& Lupo, M. P. (2009). Cosmeceuticals: practical application. Dermatologic Clinics, 27(4), 401-406.

[5] Davis, E. C., \& Callender, V. D. (2010). Postinflammatory hyperpigmentation: A review of the epidemiology, clinical features, and treatment options in skin of color. Journal of Clinical and Aesthetic Dermatology, 3(7), 20-31.

[6] Liao, A. H., Ma, W. C., Wang, C. H., \& Yeh, M. K. (2014). Penetration depth, concentration and efficiency of transdermal $\alpha$-arbutin delivery after ultrasound treatment with albumin-shelled microbubbles in mice. Drug Delivery, 27(3), 2173-2182.

[7] Rudnitskaya, A., Torok, B., \& Torok, M. (2010). Molecular docking of enzyme inhibitors: A computational tool for structure-based drug design. Biochemistry and Molecular Biology Education, 38(4), 261-265.

[8] Di Muzio, E., Toti, D., \& Polticelli, F. (2016). DockingApp: A user friendly interface for facilitated docking simulations with AutoDock Vina. Journal of Computer-Aided Molecular Design, 31(2), 213-218.

[9] Trott, O., \& Olson, A. J. (2010). AutoDock Vina: improving the speed and accuracy of docking with a new scoring function, efficient optimization and multithreading. Journal of Computational Chemistry, 31. 
455-461.

[10] Lim, Y. J., Lee, E. H., Kang, T. H., Ha, S. K., Oh, M. S., \& Kim, S. M. et al. (2009). Inhibitory effects of arbutin on melanin biosynthesis of $\alpha$-melanocyte stimulating hormone-induced hyperpigmentation in cultured brownish guinea pig skin tissues. Archives of Pharmacal Research, 32(3), 367-373.

[11] Garcia-Jimenez, A., Teruel-Puche, J. A., Ortiz-Ruiz, C. V., Berna, J., Tudela, J., \& Garcia-Canovas, F. (2016). 4-n-butylresorcinol, a depigmenting agent used in cosmetics, reacts with tyrosinase. International Union of Biochemistry and Molecular Biology, 68(8), 663-672.

[12] Kastritis, P. L., \& Bonvin, A. M. J. J. (2013). On the binding affinity of macromolecular interactions: Daring to ask why proteins interact. Journal of the Royal Society Interface, 10(79), 1-28.

[13] Boissy, R. E., Visscher, M., \& deLong, M. A. (2005). DeoxyArbutin: A novel reversible 356 tyrosinase inhibitor with effective in vivo skin lightening potency. Experimental Dermatology, 14, 601-608.

[14] Chawla, S., Kvalnes, K., deLong, M. A., Wickett, R., Manga, P. B., \& oissy, R. E. (2012). Deoxyarbutin and its derivatives inhibit tyrosinase activity and melanin synthesis without inducing reactive oxygen species or apoptosis. Journal of Drugs in Dermatology, 11, 28-34.

[15] Tanchuk, V. T., Tanin, V. O., Voyk, A. I., \& Poda, G. (2016). A new, improved hybrid scoring function for molecular docking and scoring based on AutoDock and AutoDock vina. Chemical Biology and Drug Design, 87(4), 618-625.

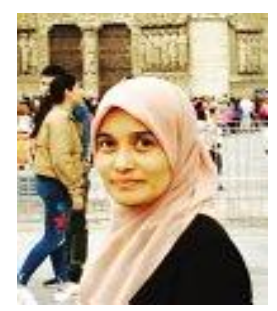

Yusnita Rifai has received the master degree in Faculty of Medicine Flinders University, Australia in 2006 under the supervision of Professor Gino Saccone. She then graduated with the Ph.D in 2011 (Chiba University, Japan), she got training from Professor Masami Ishibashi Laboratorium, Graduate School of Pharmaceuticals Science. She is oriented on the development of new candidate of drug particularly as anticancer, anti-osteoporosis, and anti-inflammation. She published more than several papers in refereed, impacted, international scientific journals.

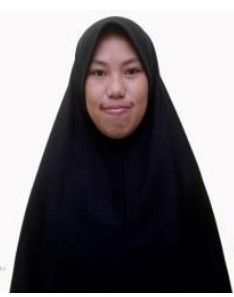

Ayu Masyita was born in July 1997. She has received a BSc degree in pharmacy from Hasanuddin University, Indonesia in March 2017. From August 2017 until now, she is postgraduate student at the Faculty of Pharmacy, Hasanuddin University, Indonesia through grant of master to doctor degree program for superior bachelor (PMDSU Scholarship Batch III) from Ministry of Science and Technology of Indonesia under the supervision of Yusnita Rifai, M.Pharm., P.hD. 